\title{
Treatment of delirium with phototherapy: a case report
}

\author{
HA Droogleever Fortuyn ${ }^{1}$, JH Schoemaker ${ }^{2}$ \\ I University Hospital Nijmegen, Department of Psychiatry, PO Box 9101, 6500 HB, Nijmegen; \\ 2 Medical Writing and Scientific Consultancy, Goirle, The Netherlands
}

(Received 20 September 1996; accepted 9 January 1997)

\begin{abstract}
Summary - A case is described where phototherapy rapidly restored sleep-wake disturbances and concurrent symptoms of delirium in a patient who did not respond to conventional treatment with haloperidol. In this case, the delirious state may have been caused by a change in metabolism as a result of sepsis. Exposition of the patient to 8,000 Lux during $1.5 \mathrm{~h}$ for 5 consecutive days was sufficient to suppress all delirious symptoms and to normalize sleep. This result may encourage the setup of systematic studies towards the extent of desynchronization between various rhythmic cycles in delirium and towards the value of phototherapy for the restoration of normal circadian rhythmicity.
\end{abstract}

delirium / phototherapy / biological rhythms / dementia / sundowning

\section{INTRODUCTION}

In delirium, symptoms of confusion and agitation can be expected to worsen during the night and early morning hours, a phenomenon called sundowning. Sundowning has also been described in cases of Alzheimer's disease (Evans, 1987; Okawa et al, 1991). Experiments have shown that phototherapy in sleep-disturbed dementia patients may normalize sleep and may have a concomitant beneficial effect on mental impairment (Okawa et al, 1991). These observations led to the use of phototherapy in a case of refractory delirium.

\section{CASE REPORT}

A 68-year-old male was hospitalized at the intensive care unit for 3 months for a disease of unknown aetiology, accompanied by sepsis. Pansinusitis was diagnosed after hospitalization and was considered to be the focus of infection. The patient also suffered from insulin-dependent diabetes mellitus with recurrent episodes of hyperglycaemia and had a serious decubitus lesion that was surgically treated. Vision was normal without signs of diabetic retinopathy. The 2 nd week after onset of hospitalization, the patient entered into a delirious state during which the sleep-wake cycle was completely disturbed. The patient slept as much during the day as at night. The delirium was characterized by frequent visual hallucinations, clouding of consciousness, and increased motor activity during the night. The nocturnal restlessness was such that the patient's arms had to be immobilized to prevent disruption of the infusion gear. Treatment with haloperidol $2.5 \mathrm{mg}$ tid for 4 weeks had some calming effect on motor activity but did not otherwise influence the delirious state.

The continuing stupor called for an alternative treatment. Haloperidol was discontinued and phototherapy was started 6 weeks after onset of delirium by exposing the patient to $8,000 \mathrm{Lux}$ in episodes of $1.5 \mathrm{~h}$ for 5 consecutive days (1930 till 2100 hours). For this purpose, a light box was used of the type SML Medilight-06 2 × 36 Watt (OT Light Systems, Benelux). During exposure, the patient was lying on his back in bed and the light box placed in front of him in transverse position. Immediately after the first exposure, the patient slept particularly well. During the night following the second exposure it was no longer deemed necessary to immobilize the patient's hands. The patient awoke from his delirious state the day after the second exposure and it was possible to com- 
municate with him for the first time in 6 weeks. The morning after the third exposure, the patient was able to put on his pyjama jacket himself, an action which he was unable to perform 6 weeks before. At completion of the phototherapy, the sleep-wake cycle in this patient was restored and all delirious symptoms had disappeared. After a period of continued observation at the ward during which some organic complications occurred (eg, epidydemitis), the patient was discharged.

At a recent return visit to the internist 2 months after discharge, cognitive functions in this patient still appeared normal.

\section{DISCUSSION}

The beneficial effect of phototherapy on disturbances in behaviour and sleep-wake cycle in this case is in agreement with the findings described in dementia patients (Okawa et al, 1991). The internal temporal order of the circadian rhythm in these patients appeared to have been lost, just as in this case, and sundowning behaviour was present. In delirious patients, the occurrence of insomnia during the night and sleepiness during the day is so predictable and often so severe, that the fundamental defect in delirium has been suggested by many to be a disturbance in the sleep-wake cycle. The case reported here may support and strengthen this hypothesis. Dementia with depressed mood could be excluded in this patient since he did not show any cognitive deficits after recovery and had not suffered from depressive episodes in any period of his life.

A deterioration in circadian rhythmicity may have various causes. It is a well accepted fact that the ontogenetic development of sleep-wake behaviour in man is dependent on an appropriate zeitgeber and intimately synchronized with other biological rhythms like body temperature and cortisol secretion. Numerous studies suggest that the suprachiasmatic nucleus in the hypothalamus plays a key role in the maintenance of stable phase relationships between the various rhythmic variables (such as sleep-wake, body temperature, and plasma cortisol concentration) in the mammalian circadian system (review by Webb and Dube, 1981). Swaab et al (1985) suggest that cell loss in this nucleus may cause disturbances in the sleep-wake cycle in demented people. Although nothing is known about anatomical and physiological connections involved between the various circadian rhythms (including sleep) and the suprachiasmatic nucleus, metabolic changes that provoke delirium may affect at the same time the functional integrity of this nucleus and cause independent phase shifts in the various rhythms or random oscillations. A strong zeitgeber, like episodes of intense light, may then rapidly restore lost synchronicity between the various circadian rhythms.

The timing of phototherapy is subject for further study. In this case, the time of application was chosen in analogy with the rationale of Satlin et al (1992), that is, that sundowning behaviour may indicate a phase advance of the sleep-wake cycle. Because agitated behaviour in delirium is usually more severe in the late evening and night, phototherapy is better not applied after 2200 hours, thus circumventing the need to fixate the patient in bed.

Successful treatment of clouded consciousness due to sepsis with phototherapy has not been described earlier in the literature. Considering the possible storage of haloperidol in the body after prolonged ( 4 weeks) use, it cannot be excluded that traces of persisting haloperidol have added to the success of this treatment in the case reported here. A systematic study would be necessary to critically evaluate the usefulness of phototherapy in the treatment of delirium.

\section{REFERENCES}

Evans LK. Sundown syndrome in institutionalized elderly. J Am Geriat Soc 1987;35:101-8

Okawa M, Hishikawa Y, Hozumi S, Hori H. Sleep-wake rhythm disorder and phototherapy in elderly patients with dementia. In: Racagni et al, eds. Biological Psychiatry, Vol I. Amsterdam: Elsevier Scientific Publishers, 1991

Satlin A, Volicer L, Ross V, Herz L, Campbell S. Bright light treatment of behavioral and sleep disturbances in patients with Alzheimer's disease. Am J Psychiatry 1992;149: 102832

Swaab DF, Fliere E, Partiman TS. The suprachiasmatic nucleus of the human brain in relation to sex, age and senile dementia. Brain Res 1985;342:37-44

Webb WB, Dube MG. Temporal characteristics of sleep. In: Aschoff J, ed. Handbook of Behavioural Neurobiology. 4. Biological Rhythms. New York: Plenum Press, 1981; 26:499-522 